\title{
Der primär-zentrale Schmerz
}

Walter Kissel

Facharzt für Innere Medizin, ehemals Gutachter MEDAS Zentralschweiz Luzern

\author{
Tiefgehende emotionale Störungen, z.B. eine Depression, können einen syndroma- \\ len Somatisierungszustand mit Schmerzen als Leitsymptom in Begleitung von neu- \\ romuskulären Funktionsstörungen mit generalisierter muskulärer Dysbalance \\ auslösen. Es findet sich eine Trias von ursächlicher emotionaler Störung, diffusen \\ "somatoformen» Schmerzen und von Störungen des muskulären Bereiches des \\ Bewegungsapparates (Verspannungen, Muskelverhärtungen, «tender points», Be- \\ wegungsblockaden).
}

\section{Einleitung}

Der Mensch ist ein hochassoziiertes, genial gestaltetes Bionetzwerk der Evolution. Es ist der Evolution für den Menschen gelungen, einen höheren Intellekt, eine verfeinerte Emotionalität mit Fähigkeit zur Empathie und Trauer und die Befähigung zur feinmotorischen Tätigkeit zu einer Ganzheit zu vernetzen. Der Mensch ist das einzige Tier, welches mit Planungsfähigkeit begabt ist und gleichzeitig den Willen entwickeln kann, Pläne durch Aktivität umzusetzen. Die Evolution hat den Menschen zur Sicherung des Lebens und Überlebens mit zwei Schmerznetzwerken ausgestattet: mit dem somatisch-nozizeptiven Schmerz, welcher körperliche Schädigungen "meldet», und mit dem isometrischen Verspannungsschmerz, welcher den Menschen zwingt, ergonomisch ungünstige Positionen zu vermeiden, um das freie Muskelspiel zu gewährleisten. Wir konstatieren beim Menschen noch eine 3. Schmerzart, für die wir oft keine Ursache finden. Man spricht dann etwa von "unklaren Schmerzen" oder "somatoformen Schmerzen» oder eventuell auch von Fibromyalgie. Schon seit der «Hysterie-Zeit» - ca. 1870-1900 - ist bekannt, dass eine

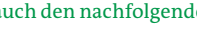

Kommentar von

Anne-Françoise Allaz. schwere emotionale Störung zu den vielfältigsten Körperfühlstörungen, von Anästhesie bis zu Hyperästhesie, und zu einer ebenfalls breiten Palette von neuromuskulären Phänomenen führen kann. Diese können sowohl Lähmungen wie auch Spasmen umfassen. Im Lehrbuch des berühmten Neurologen Hermann Oppenheim (1905) [1] schreibt der Co-Autor, welcher das Kapitel «Hysterie» bearbeitete, er habe «keine Hysterika gesehen, die nicht an Schmerzen gelitten hätte». Heute spricht man nicht mehr von «Hysterie», sondern von «dissoziativen Störungen». Der Autor konnte generell bei Gutachtensklientinnen und -klienten mit dieser Diagnose beobachten, dass hier auch die Schmerzschwelle dissoziiert war. Sigmund Freud und Josef Breuer präsentierten 1895 eindrückliche Fälle von

Die Evolution hat den Menschen zur Sicherung des Lebens und Überlebens mit zwei Schmerznetzwerken ausgestattet.

Schmerzpatientinnen mit gleichzeitigen neuromuskulären Symptomen [2]. In ihrem Buch legten sie ein psychoanalytisches Diagnose- und Therapiemodell vor. Es war ebenfalls Sigmund Freud, welcher 1895 die Angst als Ursache chronischer Schmerzen beschrieben hat [3]. In den letzten Jahrzehnten hat man erkannt, dass chronische unklare Schmerzen auch ein Symptom einer somatisierten Depression sein können [4].

\section{Patienten / Material / Methode}

Der Autor hat während 25 Jahren als vollamtlicher internistischer fallführender Gutachter der MEDAS Zentralschweiz in einem Team mit Rheumatologen und Psychiatern Schmerzpatienten begutachtet [5]. 


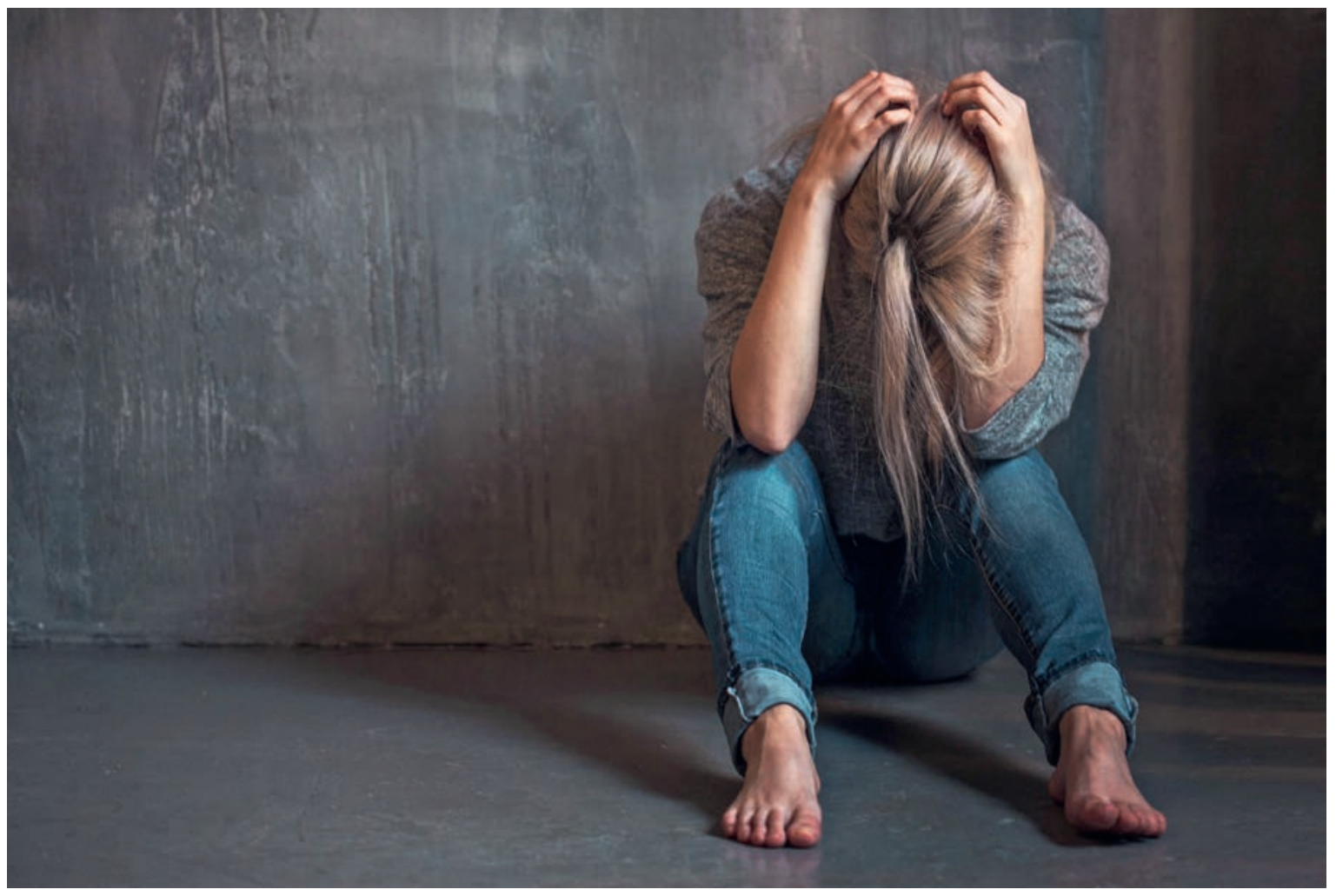

In den letzten Jahrzehnten hat man erkannt, dass chronische unklare Schmerzen auch ein Symptom einer somatisierten Depression sein können.

\section{Beobachtungen bei Gutachtens- klientinnen und -klienten}

1990 wurde zum ersten Male die «Fibromyalgie» beschrieben. Eine Kausalität des Leidens konnte noch nicht eruiert werden, wir verwendeten «Fibromyalgie» ab 1990 aber oft als Hauptdiagnose bei unklaren Schmerzen. Es fiel uns auf, wie häufig die Patientinnen (es waren ja hoch überwiegend Frauen) an psychischen Störungen litten, die wir als kausal für die Fibromyalgie-Symptomatik beurteilten. 1998 publizierten der Autor und ein MEDAS-Psychiater eine entsprechende Studie über 157 Fälle. Gutachter verfügen oft über umfangreiche Vorakten, und deshalb war uns die Aussage möglich, dass in 70\% der Fibromyalgie-Fälle, bei denen wir eine psychische Störung vorfanden, diese Störung vor Ausbruch der Schmerzen vorgelegen hatte, somit kausal für das Leiden war. Wir bezeichneten die Fibromyalgie als psychosomatische Erkrankung. Dies fand nicht ungeteilte Zustimmung, besonders nicht bei der Fibromyalgie-Gesellschaft und den Betroffenen: Sie fühlten sich psychisch stigmatisiert, in ihren Schmerzen nicht ernst genommen! Leider negieren auch die revidierten ACR-Kriterien der Fibromyalgie von 2010 eine Psychogenese der Fibromyalgie. Ab 1995 gingen wir immer häufiger auf die Diagnose «Anhaltende somatoforme Schmerzstörung» über. Die Patientenkollektive und die Ätiologien blieben naturgemäss sehr ähnlich denjenigen der Fibromyalgie. Wir fanden das Schmerzsyndrom vor allem bei der Depression, bei Angst- und Panikstörungen, bei posttraumatischen Belastungsstörungen, bei Menschen nach sexuellen Übergriffen in der Kindheit, bei dissoziativen Störungen, bei Borderline-Störungen.

Aufschlussreiche Erkenntnisse konnte der Autor aus der Beobachtung von Klientinnen und Klienten mit Depressionen und bipolaren Störungen gewinnen. Man kann ja die Depression mit ihrer gedrückten Stimmung, mit ihrer starren Emotionalität, mit ihrer Erschöpfung, mit ihren verlangsamten motorischen Abläufen, mit ihren diffusen Körperschmerzen und mit der Unfähigkeit, einen Willen aufzubringen, etwas zu tun, auch als «Energiemangel-Krankheit» bezeichnen. Ganz anders imponiert dann ein manischer Schub: Die Stimmung ist gehoben, die Energie und Aktivitäten scheinen unerschöpflich zu sein, Schmerzen werden kaum wahrgenommen. Eine Klientin wurde während einer langjährigen Depression mit dem körperlich-klinischen Bild einer Fibromyalgie über Jahre hinweg physiotherapeutisch behandelt; eines Tages stellten sich zusätzliche manische Schübe ein, die sogar dann in eine rapid-cycling bipolare Psychose 
übergingen. Die Klientin berichtete dem Autor, dass sie, noch während am Vortag von der Physiotherapeutin wegen ihrer Schmerzen behandelt, plötzlich von der Lust zum Tanzen ergriffen worden sei; sie sei in ein Dancing gegangen, habe die ganze Nacht durchgetanzt, die Schuhe seien zerfetzt gewesen, Blut sei ausgetreten. Als sie nach Hause kam, sei gerade ihr Mann aufgestanden und habe entsetzt auf ihre Füsse gestarrt - sie selber habe überhaupt nichts gemerkt!

Der Autor konnte ganz allgemein beobachten, dass viele psychische Erkrankungen mit der Trias emotional-psychische Störung, unklare Körperschmerzen und neuromuskuläre Dysbalance einhergehen können. Es entspricht auch der Erfahrung des Autors, dass besonders auffällige Schmerz- und Klagebilder auf sexuellen Übergriffen in der Kindheit basieren können. Bei solchen Klagen hat der Autor immer gefragt: Was ist in Ihrer Kindheit passiert? Sind Sie Opfer von Übergriffen geworden? Es kam mehrmals zur Auslösung einer Schockreaktion. Eine Klientin sagte mir: Das weiss nicht einmal mein Mann.

\section{Modellvorstellungen des Autors: die Auslösung zentraler Schmerzen}

Bei der bipolaren Psychose oszillieren sowohl die Emotionslage, der Muskeltonus wie auch die Schmerzschwelle um die Normalbereiche des gesunden Menschen, es oszilliert ebenso die Energiebildung. Auch andere psychische Störungen können solche Oszillationen aufweisen. Der Autor kommt zum Schluss, dass die Netzwerke der Emotionalität durch eine «Energiezentrale» bedient werden, welche gleichzeitig auch Energie in den neuromuskulären Basaltonus und in die Stabilisierung der Schmerzschwelle liefert. Eine tiefgehende emotionale Störung kann zu einem Abfall des zentralen Energieflusses in den Netzwerken der Emotionalität führen. Unter anderem wird die Schmerzschwelle destabilisiert. Folgerung: Bei jeder unklaren Schmerzkrankheit muss eingehend nach einer möglichen psychischen Ursache gesucht werden.

Der Autor vermutet eine evolutionäre Grundlage in folgender Gegebenheit: in der Fähigkeit des Menschen zur Feinmotorik. Feinmotorik benötigt Planungsfähigkeit (= intellektueller Bereich), Willensbildung (= emotionaler Bereich), motorische Steuerung und sensible Rückmeldungen aus der Peripherie zur Adaptation der Motorik. Ein Energieabfall im «Zentrum» oder eine Blockade (z.B. bei dissoziativen Störungen) löst die jeweilige syndromale Symptomatik aus. Der Autor vermutet gleichzeitig, dass die «Energiezentrale» für die «Bedienung» aller Wirkbereiche mit einem gemeinsamen «Botenstoff» arbeitet, am ehesten mit einem körpereigenen Morphin (Endorphin). Dies könnte die Tatsachen erklären, dass die Opiate einerseits unsere stärksten Schmerzmittel gegen Akutschmerzen darstellen, andererseits aber bei Nicht-Schmerzleidenden die Stimmung heben, zur Euphorisierung führen können. Fällt der Wirkspiegel ab, nehmen die Schmerzen wieder zu, und die Euphorie wird zur Dysphorie. Opiate können somit die Schmerzschwelle wie auch den Zustand der Emotionalität oszillieren lassen.

Freud und Breuer konnten schon 1895 klar nachweisen, dass psychische Belastungen, Konflikte und emotionale Traumata die Symptome der Hysterie auslösen konnten. Für ihre Anamneseerhebung, auf der Suche nach auslösenden Ursachen, wendeten sie in Einzelfällen pro Patientin bis zu mehrere Hundert (!) Stunden auf. Der Nachweis der Psychogenese des Leidens konnte dadurch erbracht werden, dass die Aufarbeitung der Traumata im Rahmen der Anamnese zum langsamen Rückgang, bis hin zur Ausheilung der Symptome führte - die Psychoanalyse war geboren! Der Neurologe H. Oppenheim [1] bezeichnete 1905 die Schmerzen der Hysterie als «Schmerzhalluzinationen», somit klar als zentrale Schmerzen.

Der Terminus "primär-zentraler Schmerz» stammt vom Autor, unter anderem aus folgenden Überlegungen heraus: Es wird in der heutigen Literatur immer wieder von "zentralisierten» Schmerzen geschrieben. Meistens ist damit eine Schmerzchronifizierung durch ein «Schmerzgedächtnis» gemeint, das heisst, dass ein anfänglich somatisch-nozizeptiver Schmerz durch das «Schmerzgedächtnis» perpetuiert wird. Es wird somit von einer "sekundär-zentralen Schmerzgenese» ausgegangen. Die Zielrichtung des Autors geht aber in diametral anderer Richtung! Die Erkenntnisse der berühmten Ärzte um 1890-1905 sind nicht mehr im «Kollektivgedächtnis der modernen Medizin». Der Autor will hier klar einen Gegenpol setzen und zum Rückgriff auf alte, aber durchaus nicht veraltete Erkenntnisse von Psychiatern und Neurologen um die Jahrhundertwende 1900 anregen.

Bildnachweis

๑) Kriscole | Dreamstime.com (Symbolbild)

Literatur

1 Oppenheim H. Lehrbuch der Nervenkrankheiten für Ärzte und Studierende. 1. Band. Berlin: S. Karger; 1905.

2 Freud S, Breuer J. Studien über Hysterie (1895). In: Fischer Taschenbuch, 1991. Dritte, korrigierte Auflage 1997.

3 Freud S. Über die Berechtigung, von der Neurasthenie einen bestimmten Symptomenkomplex als «Angstneurose» abzutren nen (1895 [1894]). In: Freud - Studienausgabe Buchclub Ex Libris Zürich 1977.

4 Delini-Stula A, Lehtinen A, Holsboer-Trachsler E. Diagnostische und therapeutische Bedeutung von Schmerzsymptomen der Depression. Schweizer Archiv für Neurologie und Psychiatrie 157.6/2006.

5 Kissel W. Das somatoforme Schmerzsyndrom. Schweiz Ärzteztg. 2018;99(23):768-71.
Walter Kissel

walter.kissel[at]bluewin.ch 